\title{
In vivo mechanisms by which tumors producing thrombospondin 1 bypass its inhibitory effects
}

\author{
Stéphanie Filleur, ${ }^{1}$ Olga V. Volpert, ${ }^{2,3}$ Armelle Degeorges, ${ }^{1}$ Carole Voland ${ }^{4}$ Frank Reiher, ${ }^{2,3}$ \\ Philippe Clézardin, ${ }^{4}$ Noël Bouck, ${ }^{3,5}$ and Florence Cabon ${ }^{1,6}$ \\ ${ }^{1}$ Institut André Lwoff, Centre National de la Recherche Scientifique UPR 9079, 94801 Villejuif, France; ${ }^{2}$ Department of \\ Urology, and ${ }^{3}$ Robert H. Lurie Comprehensive Cancer Center, Northwestern University Medical School, Chicago, Illinois \\ 60611, USA; ${ }^{4}$ Institut National de la Santé et de la Recherche Médicale U403, Faculté de Médecine Laënnec, 69372 Lyon, \\ France; ${ }^{5}$ Department of Microbiology-Immunology, Northwestern University Medical School, Chicago, Illinois 60611, USA
}

Thrombospondin 1 (TSP1) is a multifunctional protein able to activate TGF $\beta$ and to inhibit angiogenesis in vivo. Although usually thought of as an inhibitor of tumor growth, TSP1 may sometimes be present at high levels during tumor progression, suggesting that tumors can eventually overcome their anti-tumor effects. Using a tet-repressible expression system, we demonstrate that murine TSP1 delayed the onset of tumor growth when produced in the tumor bed by rat fibrosarcoma tumor cells or by stromal fibroblasts coinjected with unmodified C6 glioma tumor cells. Yet upon prolonged exposure to TSP1, tumors came to grow at the same rate in the presence as in the absence of TSP1 and transplantation experiments showed that they had become insensitive to inhibition by TSP1 in both syngeneic and immune compromised hosts. Tumor resistance to TSP1 developed as a result of the in vivo outgrowth of pre-existing tumor cell variants that (1) secreted increased amounts of angiogenic factors that counterbalanced the inhibitory effect of TSP1 on neovascularization and (2) grew more efficiently in the presence of TSP1-activated TGF $\beta$. These results indicate that prolonged and continuous local delivery of a single multifunctional angiogenesis inhibitor like TSP1 to fast-growing tumors can lead to tumor resistance in vivo by fostering the outgrowth of subpopulations that are a by-product of the genetic instability of the tumor cells themselves.

[Key Words: Angiogenesis; inhibition; cancer; TGFß; resistance; tetracyclin]

Received November 22, 2000; revised version accepted April 5, 2001.

Thrombospondin-1 (TSP1) is a 450-kD extracellular matrix glycoprotein secreted by many cell types (Bornstein 1995) that inhibits both tumor cell growth and metastasis formation in vivo (Boukamp et al. 1997; Sheibani and Frazier 1995; Volpert et al. 1998; Weinstat-Saslow et al. 1994). Tumor cell lines usually express very low levels of TSP1 (Mettouchi et al. 1994; Salnikow et al. 1994; Sheibani and Frazier 1996; Slack and Bornstein 1994; Tikhonenko et al. 1996). Re-expression of TSP1 in such tumor cells suppresses their tumorigenicity in vivo (Boukamp et al. 1997; Sheibani and Frazier 1995; Weinstat-Saslow et al. 1994). In addition, in nude mice, secretion of TSP1 by a distant HT1080 fibrosarcoma has been shown to impair the growth of B16/F10 melanoma lung metastases (Volpert et al. 1998).

The inhibitory effect of TSP1 on tumor growth and metastasis formation results in part from its ability to block angiogenesis. Indeed, TSP1 inhibits basic fibro-

${ }^{6}$ Corresponding author.

E-MAIL fcabon@vjf.cnrs.fr; FAX +33-(0)-1-49-58-33-07.

Article and publication are at www.genesdev.org/cgi/doi/10.1101/ gad.193501. blast growth factor-induced angiogenesis in a rat cornea model (Good et al. 1990; Tolsma et al. 1993), exogenously added TSP1 blocks the ability of cultured capillary endothelial cells to organize into cords (Tolsma et al. 1997), and down-regulation of endothelial cell TSP1 enhances angiogenesis in vitro (Canfield and Schor 1995; DiPietro et al. 1994; Iruela-Arispe et al. 1991; Tolsma et al. 1997). Inactivation of the TSP1 gene in mouse results in hypervascularization in dermis and pancreas (Crawford et al. 1998). In humans, anti-angiogenic properties of TSP1 have been reported in bladder cancer (Campbell et al. 1998), melanoma (Zabrenetzky et al. 1994), and gastro-intestinal tumors (Morelli et al. 1998). Conversely, in colorectal cancer, high TSP1 level in plasma is associated with increased angiogenesis (Yamashita et al. 1998) and with lymph node metastasis in gallbladder cancers (Ohtani et al. 1999). In breast cancer, both pro- and antiangiogenic correlations to TSP-1 are reported (Bertin et al. 1997; Clézardin et al. 1993; Morelli et al. 1998; Zabrenetzky et al. 1994). These apparent conflicting results suggest that some tumors may develop the ability to counteract the inhibitory effect of TSP1.

Similar phenomena have been observed with other 
anti-angiogenic agents. A variety of tumors, including prostate, urinary bladder, and colon carcinomas, have been identified that are able to grow well while secreting uncharacterized angiogenesis inhibitors at levels sufficient to control the growth of distant second tumors (Chen et al. 1995; Prehn 1993). In some cases the antiangiogenic activity could be assigned to a specific molecule such as angiostatin in Lewis lung carcinoma (O'Reilly et al. 1994), thrombospondin in the fibrosarcoma HT1080 (Volpert et al. 1998), or the serpin antithrombin in a small cell lung cancer (Chen et al. 1995). It has been hypothesized but never directly shown that these tumors counterbalance their own secretion of inhibitors by local overproduction of molecules that stimulate angiogenesis (Chen et al. 1995; Prehn 1993; Volpert et al. 1998).

Using a tet-repressible expression system for TSP1, we demonstrate here how the ability to bypass the inhibitory effects of an anti-angiogenic agent develops in vivo. Although TSP1 expression by tumor cells or by their stroma strongly inhibited early steps of tumorigenesis, upon continuous local delivery of TSP1, tumor cells that were able to bypass the inhibitory effects of TSP1 were selected. Analysis of such cells revealed two cooperative mechanisms that contributed to the acquisition of this trait.

\section{Results}

TSP1 inhibits the onset of tumorigenesis but has only a transient effect on developed tumors

We have shown previously that rat fibroblasts transformed by c-Jun are highly tumorigenic in vivo and their endogenous TSP1 expression is significantly repressed (Dejong et al. 1999; Mettouchi et al. 1994). To study the functions of TSP1 during tumor progression, we introduced a tet-repressible bidirectional vector /Gossen and Bujard 1992) to express murine TSP1 and luciferase in cJ4 cells (Fig. 1A). The derepression of TSP1 and luciferase expression in this system is achieved, in vitro and in vivo, in the absence of any drug, thus avoiding any influence of the drug on the TSP1-induced phenotype (Fig. 1B). TSP1 expression was repressed during selection to avoid bias, a particular concern when working with genetically unstable tumorigenic cells. Three representative clones, JT4, JT8, and JT13, were chosen and further analyzed. In vitro, doxycycline (dox) withdrawal induced the synthesis and secretion of TSP1 in these clones at levels comparable to those of nontransformed rat fibroblasts (Fig. 1C; data not shown). TSP1 re-expression, in the absence of dox, did not modify the morphology or adherence of the cells or the growth rate, maximal cell density, or anchorage-independent growth of either clone as has been noted previously in other tumor types expressing exogenous TSP1 (Streit et al. 1999; WeinstatSaslow et al. 1994).

When JT4, JT8, JT13 cells or a $(1: 1: 1)$ mix of these clones were injected subcutaneously into syngeneic Fisher rats, tumor take was $100 \%$ in both untreated (dox-) and in dox-treated (dox + ) animals. Dox repression of the introduced promoter was effective in vivo, for luciferase activity in tumor homogenates increased $5.1 \pm 0.6$-fold when tumors grown in dox-fed animals were compared to those grown in untreated animals and TSP1 secretion could be detected immunohistochemically in the absence of dox (Fig. 2A). A reproducible increase of the latency periods by 3-5 d was observed in the untreated group expressing TSP1. Development of the JT8 tumors was monitored during 4 wk (Fig. 1D); when harvested, tumors formed by JT8 cells from dox-fed animals, in which TSP1 was repressed, weighed $8.56 \pm 3.2 \mathrm{~g}$ whereas the average weight of tumors from JT8 expressing TSP1 was markedly reduced $(1.7 \pm 1.0 \mathrm{~g})$. The mean reduction in tumor volume calculated from four independent experiments was $81.9 \pm 9.4 \%$. Comparable results were observed in tumors from JT4 and JT13 individual clones (data not shown). TSP1 expression induced a $40 \%$ reduction in the volume of tumors that formed when a mix of the three former clones was injected (Fig. 1E). TSP1 control of tumor growth was independent of the immune system as a $65 \%$ decrease in tumor volume was seen when the JT8 cells were injected in nude mice (Fig. 1F). The growth of the tumors derived from cJ4 parental cells, or from cJ4 cells harboring a dox-repressible luciferase gene, was not affected by dox treatment, indicating that the tet system per se and the dox treatment had no effect on tumor growth (data not shown).

The dramatic reduction in the size of tumors grown when TSP1 was expressed seemed to be the result of an initial delay in tumor development. Once tumors established themselves, those expressing TSP1 grew at the same rate as tumors where TSP1 was repressed (Fig. $1 \mathrm{D}, \mathrm{E}$, insets), as if cells expressing TSP1 had become resistant to its inhibitory effects.

Extrapolation at the origin of the logarithmical trendline of the tumor growth curves allows to estimate the percentage of injected cells that will develop as a tumor in the presence of TSP1 as compared to that in the absence of TSP1, taken as $100 \%$. This percentage was in the $10 \%$ range for individual clones (Fig. 1D, inset; data not shown) and above $50 \%$ when a mix of clones was injected (Fig. 1E, inset). In addition, the activation of TSP1 expression in JT8 tumors by dox withdrawal at any time before day 14, when tumors become palpable, inhibited the tumor growth to the same extent /data not shown). In contrast, TSP1 re-expression upon dox withdrawal after day 18, once tumors were well established, induced only a transient reduction in tumor volume (Fig. 1F).

\section{Fibrosarcomas develop the ability to bypass the inhibitory effects of TSP1}

To determine if tumor resistance had indeed developed in vivo in the presence of TSP1, cells from tumors that had formed in the presence or in the absence of TSP1 were recovered and propagated in culture (Fig. 1A). More than $90 \%$ of all the tumor cells retained the ability to grow in selective medium and continued to be sensitive 
a

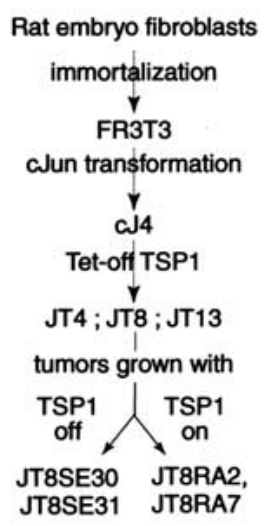

b

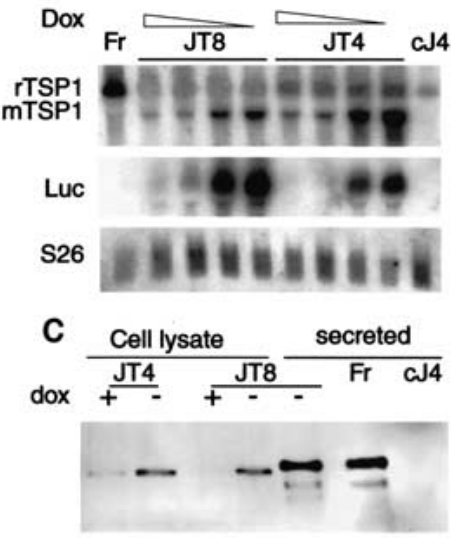

d



$\mathrm{h}$ e

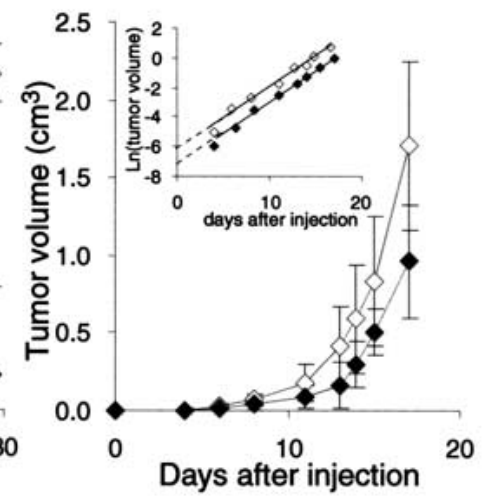

f

g
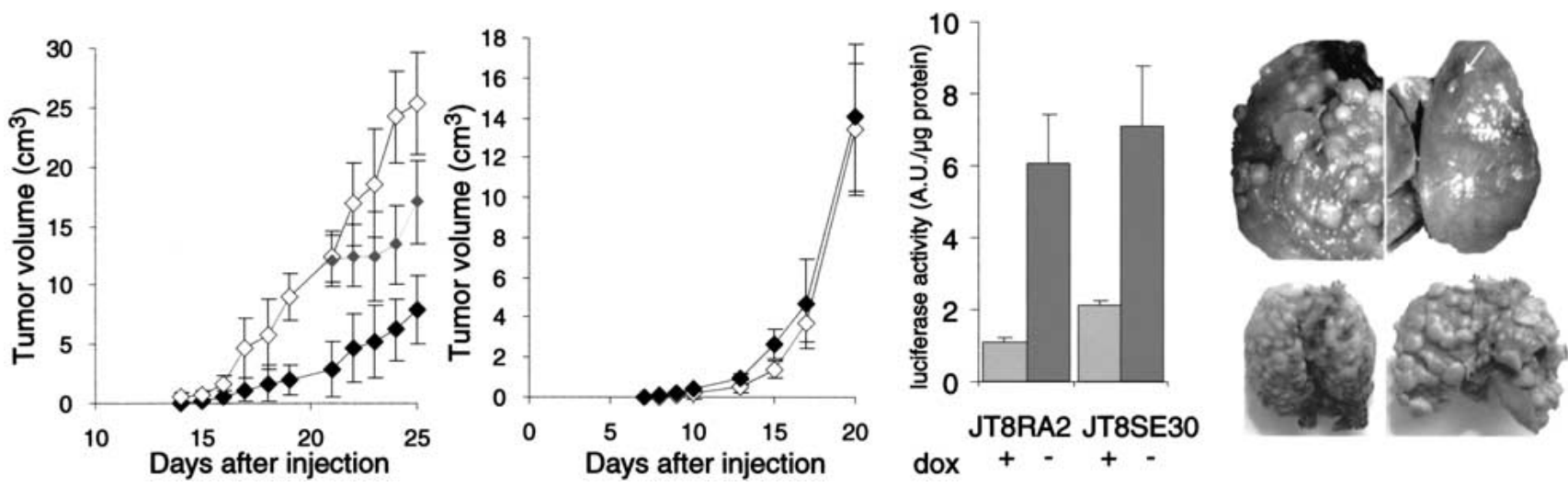

Figure 1. TSP1 effect on JT8 sarcomas in vivo. (A) Lineage of the cells used. $(B)$ Northern blot analysis of TSP1 mRNA content in FR3T3 (Fr), cJ4 cells, JT4, and JT8 inducible clones grown in the presence of decreasing (doxycycline) dox concentrations, from 100, $1,0.1$, to $0 \mathrm{ng} / \mathrm{mL}$. S26 mRNA is used as a loading control. (C) Western blot analysis of TSP1 content in cell lysates or serum-free conditioned media of FR3T3 (Fr), cJ4, JT4, and JT8 cells grown in the presence or absence of dox as indicated. Cells $\left(10^{6}\right)$ were injected subcutaneously to syngeneic Fisher rats or Swiss nude mice receiving $(\diamond)$ or not receiving $(\diamond)$ dox in drinking supply. $(D)$ Volume of JT8 tumors in rats (mean \pm SEM). Inset: Logarithmic conversion of the growth curves. (E) Volume of tumors from a mix of 3 fibrosarcoma clones in nude mice. Inset: Logarithmic conversion of the growth curves. $(F)$ Volume of JT8 tumors in nude mice receiving dox continuously $(\diamond)$, until day $18(\diamond)$, or no dox $(\diamond) .(G)$ Tumor growth of JT8RA2 cells in Fisher rats. $(H)$ Luciferase activity resulting from the bidirectional TSP1-luciferase tet-repressible vector expression in homogenates of JT8RA2 or JT8SE30 tumors collected in animals treated with dox or not treated as indicated (relative luminometric units / $\mu$ g protein, mean \pm SEM, 4 animals/ group). (I) Lungs from nude mice injected intravenously with $10^{6} \mathrm{JT} 8$ (upper panels) or JT8RA2 cells (lower panels) and receiving dox (left panels) or not (right panels). Six mice were injected in each group and aspects of the lungs were similar.

to dox inhibition of TSP1 production (Fig. 2D). But, when tested in vivo, both in syngeneic and immune compromised rodents, cells from tumors that had originally grown in the presence of TSP1 (clones JT8RA2 and JT8RA7) were resistant to its inhibitory effects (Fig. 1G; data not shown). In contrast, second generation tumors, developing from cells recovered from the tumors originally grown in absence of TSP1 (clone JT8SE30), remained sensitive in that their growth was inhibited by $66 \%$ upon expression of TSP1 (data not shown). Dox repression remained effective in vivo in both sensitive and resistant tumors for those growing without dox expressed three- (JT8SE30) to fivefold ( JT8RA2) as much luciferase as those growing in the presence of dox (Fig. $1 \mathrm{H})$, and increased TSP1 could be detected on Western blots of plasma drawn from the untreated tumor-bearing animals (data not shown).

TSP1 expression by JT8 cells in the absence of dox also induced a large reduction in the number of macroscopic metastases that formed when the cells were injected intravenously (Fig. 1I, upper panels). In contrast, injected JT8RA2 cells formed lung metastases as well in dox treated or untreated animals (Fig. 1I, lower panels).

\section{Resistant tumor cells counterbalance anti-angiogenic properties of TSP1}

To determine what might be responsible for the development of resistance to the inhibition of tumor growth by TSP1, we first examined angiogenesis, as TSP1 is a 
Filleur et al.
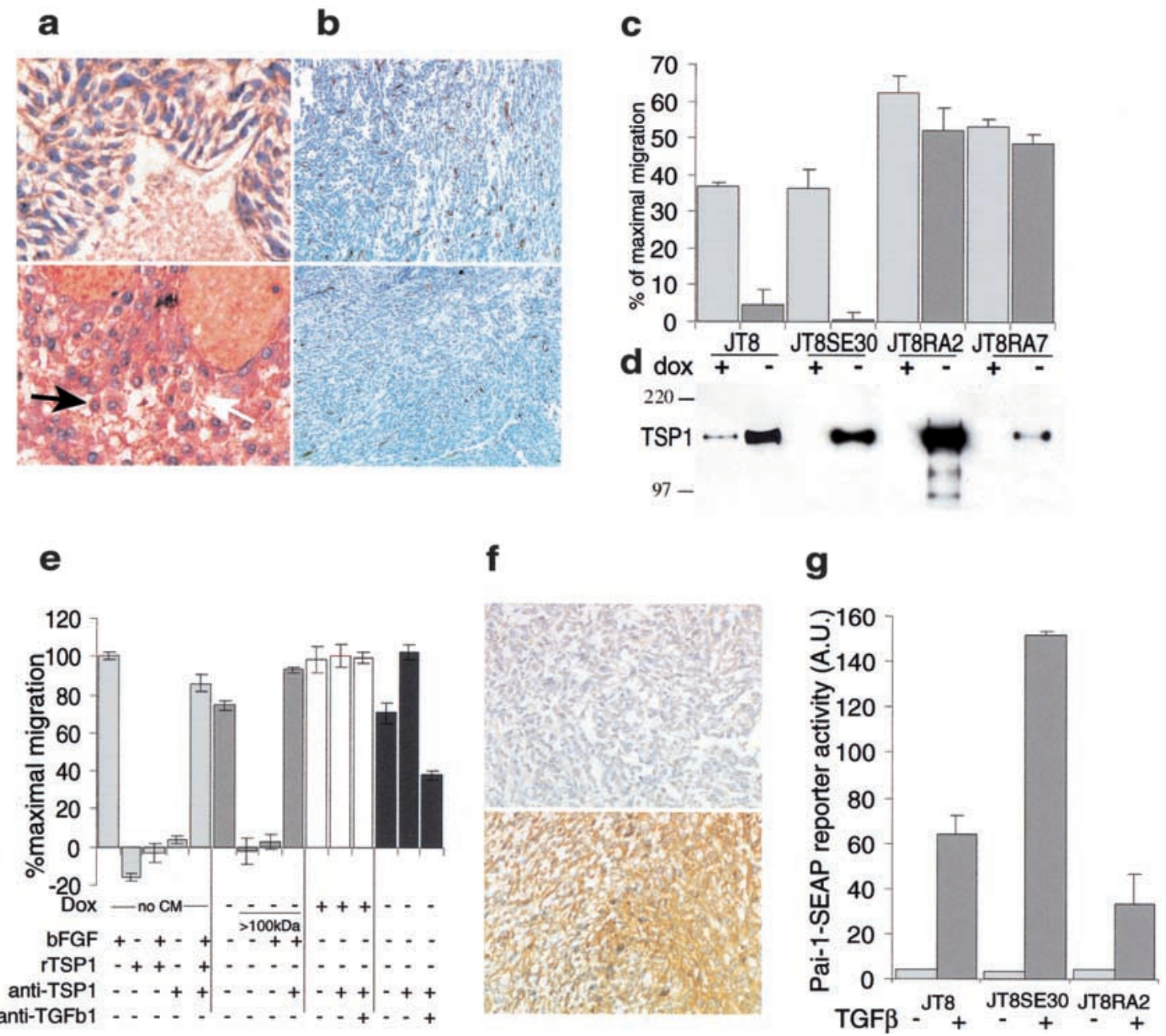

Figure 2. Resistance to inhibitory effects of TSP1. (A) TSP1 immunostaining of the sections of harvested JT8 tumors in rats. Upper panels, dox + tumors, lower panels, dox - tumors. Expression of TSP1 in the cytosol (black arrow) and secretion in the matrix (white arrow) is shown. (B) CD31 staining of endothelial cells on tumor sections as in $A$. $(C)$ Endothelial cell migration toward serum-free conditioned media from parental cells (JT8), from TSP1 sensitive cells recovered from tumors grown with TSP1 off (JT8SE30) and from TSP1 resistant cells recovered from tumors grown in the presence of TSP1 (JT8RA2 and JT8RA7) that were cultured in vitro in the presence of dox to repress TSP1 $(\square)$ or in the absence of dox $(\square)$ and adjusted to $1 \mu \mathrm{g} / \mathrm{mL}$ for total protein content. $(D)$ Western blot of the TSP1 content in the media used in migration assays in C. (E) Migration of endothelial cells in the absence of CM or in the presence of CM from JT8RA2 cells, grown in absence of doxycycline and unfractionated or dialyzed using a $100 \mathrm{kD}$ cut off $(>100 \mathrm{kD})$. Media were assayed alone, along with bFGF, with bFGF and anti-TSP1 blocking antibodies or with anti TGF $\beta$ blocking antibodies as indicated below. The results are expressed as in $C$. $(F)$ Immunolabeling of active forms of TGF $\beta$ performed on sections of JT8RA2 tumors in nude mice. Upper panels, dox + tumors, lower panels, dox- tumors. $(G)$ Activation of a transfected PAI-1-SEAP reporter construct in JT8, JT8RA2, and JT8SE30 cells upon addition of recombinant hTGFß1.

potent inhibitor of neovascularization. As expected, the expression of TSP1 in parental tumors was accompanied by a $57 \%$ reduction in microvascular density (Fig. $2 \mathrm{~B}$; Table 1). A similar difference was seen in tumors formed by a sensitive clone, JT8SE30, but in tumors derived from resistant clone JT8RA2, vessel density was no longer reduced in the presence of TSP1 (Table 1). To identify the mechanisms involved in this resistance to the anti-angiogenic effects of TSP1, both sensitive and resistant cells were grown in culture and the angiogenic activity of their serum-free conditioned media measured using the ability to induce chemotaxis of capillary endothelial cells as an assay. Parental JT8 and sensitive
JT8SE30 cells were angiogenic only when TSP1 secretion was suppressed by dox, whereas resistant clones JT8RA2 and JT8RA7 were angiogenic regardless of the expression of TSP-1 (Fig. 2C). This difference was not due to a difference in the amount of TSP1 secreted (Fig. 2D), nor could it be attributed to a mutation that had inactivated the TSP1 secreted by resistant cells. A dialyzed high molecular weight fraction of these media, that retained TSP1 but was depleted of stimulatory activity, was still able to inhibit the bFGF-induced migration of endothelial cells and was sensitive to neutralizing anti-TSP1 antibodies (Fig. 2E). Rather, the resistant differed from the sensitive cells in that they had increased their produc- 
Table 1. Angiogenic profile of tumors growing with or without TSP1

\begin{tabular}{|c|c|c|c|c|c|c|c|}
\hline \multirow[b]{3}{*}{ Tumor cells } & \multirow{3}{*}{$\begin{array}{c}\text { Tumor } \\
\text { phenotype }\end{array}$} & \multirow{2}{*}{\multicolumn{2}{|c|}{$\begin{array}{c}\begin{array}{c}\text { Tumor vessels/ } \\
\text { high power field }\end{array} \\
\text { Tumors grown with }\end{array}$}} & \multicolumn{2}{|c|}{ Secreted angiogenic activity } & \multirow{2}{*}{\multicolumn{2}{|c|}{$\begin{array}{c}\begin{array}{c}\text { TGF } \beta 1 \text { effect } \\
\text { on cell growth }\end{array} \\
\text { Doubling time }(\mathrm{h})\end{array}$}} \\
\hline & & & & \multirow{2}{*}{$\begin{array}{c}\text { ED } 50^{\mathrm{b}} \\
(\mu \mathrm{g} \text { protein } / \mathrm{mL})\end{array}$} & \multirow{2}{*}{$\begin{array}{c}\text { VEGF } \\
\text { (pg/ug protein) }\end{array}$} & & \\
\hline & & TSP1 off & TSP1 on & & & control & $+\mathrm{TGF} \beta$ \\
\hline \multicolumn{8}{|l|}{ Fibrosarcoma } \\
\hline JT8 & TSP1-sensitive & $31.6 \pm 7.2^{\star}$ & $13.4 \pm 9^{\star}$ & 0.60 & $44.0 \pm 7.6$ & 19.2 & 23.7 \\
\hline JT8SE31 & TSP1-sensitive & N.D. & N.D. & 0.61 & $30.3 \pm 11.2$ & N.D. & N.D. \\
\hline JT8SE30 & TSP1-sensitive & $17.9 \pm 1.6$ & $10.3 \pm 0.7$ & 0.60 & $28.4 \pm 1.6$ & 23.3 & 29.6 \\
\hline JT8RA2 & TSP1-resistant & $21.4 \pm 6.5$ & $23.9 \pm 9.4$ & 0.13 & $65.6 \pm 2.7$ & 20.9 & 21.0 \\
\hline T8RA7 & TSP1-resistant & N.D. & N.D. & 0.20 & $92.6 \pm 6.7$ & N.D. & N.D. \\
\hline \multicolumn{8}{|l|}{ Glioblastoma } \\
\hline C6 parent & TSP1-sensitive & $38 \pm 11^{\star \star}$ & $24 \pm 9^{\star \star}$ & 0.60 & $3.8 \pm 0.18$ & 40.3 & 40.4 \\
\hline C6S1 & TSP1-sensitive & $14.7 \pm 6.6$ & $10.6 \pm 2.9$ & 0.89 & $4.2 \pm 0.32$ & 34.3 & 34.3 \\
\hline C6R2 & TSP1-resistant & $12.2 \pm 2.0$ & $13.3 \pm 1.4$ & 0.21 & $6.2 \pm 0.12$ & 31.4 & 25.5 \\
\hline
\end{tabular}

ablood vessel density in 2-3 $\mathrm{cm}^{3}$ tumors. The tumors labeled * were above $10 \mathrm{~cm}^{3}$; the tumors labeled ** were not volume-matched.

${ }^{\mathrm{b}}$ Measured as $\mu \mathrm{g}$ secreted protein necessary to achieve $50 \%$ of maximum induction of angiogenesis in a migration assay. In the case of the fibrosarcoma, samples were tested in the presence of antibodies neutralizing TSP1 activity.

${ }^{c}$ Cells were grown in medium containing $10 \%$ (fibrosarcoma) or $2 \%$ (glioblastoma) serum in the presence of TGF $\beta$ or vehicle as indicated.

N.D., Not determined.

tion of pro-angiogenic factors. When the total stimulatory angiogenic activity was quantified by measuring the amount of secreted protein necessary to induce half maximal migration of capillary endothelial cells, resistant clones were found to release at least 3 times the stimulatory activity of sensitive clones (Table 1). Experiments with neutralizing antibodies demonstrated that vascular endothelial growth factor (VEGF) was one of the major angiogenic factors secreted by these cells (data not shown) and a parallel increase in total secreted VEGF was detected in resistant cells (Table 1).

\section{Loss of growth inhibition of resistant tumor cells} by $T G F \beta 1$

In addition to its ability to inhibit angiogenesis, TSP1 is a potent in vivo activator of TGF $\beta$ (Crawford et al. 1998), a cytokine that can inhibit or stimulate the growth of tumor cells depending on cellular context (Massague et al. 2000). When TSP1 was turned on by dox withdrawal, increased active TGF $\beta$ could be detected in the tumor bed in vivo in JT8 parental, JT8SE30 TSP1-sensitive as well as in JT8RA2 TSP1-resistant tumors, using antibodies that recognize only activated TGF 3 (Fig. 2F). An increase in active TGF $\beta$ could also be detected in vitro, when TSP1 was turned on, for the angiogenic activity of conditioned media was partially decreased in the presence of antibodies that neutralized TGF $\beta$ (Fig. 2E). To determine if the tumor cells were sensitive to TGF $\beta$, growth was measured in the presence of dox to suppress TSP1 and TSP1-dependent activation of TGF $\beta$ (Table 1). Recombinant TGF $\beta 1$ partially inhibited the growth of parental JT8 and TSP1-sensitive JT8SE30 but not of TSP1-resistant JT8RA2 cells. In all clones, TGF $\beta$ activated a transfected PAI-1-alkaline phosphatase reporter vector to a similar extent (Fig. 2G), indicating that both sensitive and resistant cells had a functional TGF $\beta$ type II receptor.
Resistance to TSP1 inhibition also develops in C6 glioblastoma via similar mechanisms

To demonstrate that the TSP1 resistance developed by the JT8 fibrosarcoma is not unique to this tumor and is not due to a TSP1 mutation in the tumor cells, another system was developed. The tet-repressible TSP1 expression system was introduced into nontumorigenic NIH3T3 mouse fibroblasts that expressed endogenous TSP1 at low levels. A dox-repressible clone (NT26) was then mixed in a 10:1 ratio with C6 rat glioblastoma cells and this mix injected subcutaneously into nude mice. Expression of TSP1 from these "stromal" cells in the absence of dox for $25 \mathrm{~d}$ induced a mean reduction of tumor volume by $75 \%$ (Fig. 3A). Linearization of the growth curves indicated that more than $50 \%$ of the C6 cells were able to contribute to tumors in the presence of TSP1 (Fig. 3A, inset). As with the fibrosarcomas, TSP1 delayed the onset of the tumor but did not modify its growth rate. Immunostaining of the dissected tumors harvested at the end of the experiment confirmed that TSP-1 was indeed expressed in C6/NT26/dox- tumors and repressed in dox+ tumors (Fig. 3B). Fibroblastic NT26 cells were also detectable histologically at this time, although no NT26 cells could be grown out in selective medium in culture from either dox+ or doxtumors. Similar results were obtained when a "stromal" expression of TSP1 was triggered with a comparable experimental model in cJ4 fibroblastic tumors (data not shown).

As with the fibrosarcoma, expression of TSP1 in C6 glioblastoma resulted in a reduction in blood vessel density by $36 \%$ (Fig. 3C; Table 1). C6 cells were recovered from tumors in which TSP1 expression by NT26 cells had been repressed (clone C6S1) and from tumors in which TSP1 had been expressed (clone C6R2) and tested for resistance to TSP1 inhibition by coinjection with fresh NT26 cells. The NT26/C6S1 tumors were still in- 
Filleur et al.


\section{$\mathbf{f}$}

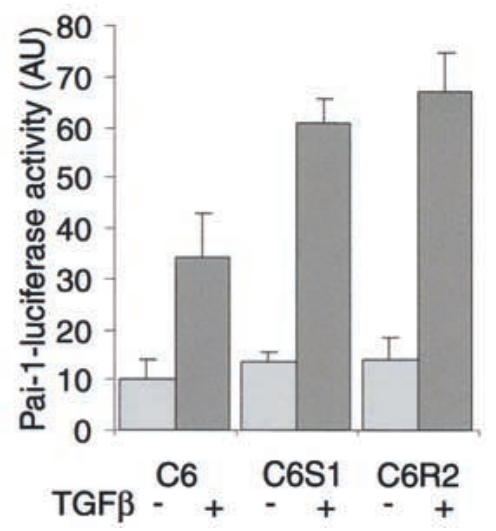

Figure 3. TSP1 effects on C6 gliomas in vivo. (A) C6 glioma cells $\left(10^{5}\right)$ were co-injected into nude mice with $10^{6}$ NT26 nontumorigenic TSP1-repressible fibroblasts and tumor volume monitored for $25 \mathrm{~d}$ in dox-treated $(\diamond)$ or untreated $(\diamond)$ nude mice. Inset: Logarithmic conversion of the growth curves. Immunostaining for TSP1 $(B)$, CD31 $(C)$, or active TGF $\beta(D)$ of the sections of harvested tumors from experiment in A. Upper panels, dox + tumors, lower panels, dox-tumors. (E) Tumor growth of C6R2 cells co-injected with fresh NT26 cells, in dox-treated $(\diamond)$ or untreated $(\diamond)$ nude mice. $(F)$ Activation of a transfected PAI-1-luciferase reporter construct in C6, C6S1, or C6R2 cells upon addition of recombinant hTGFß1.

hibited by TSP1 expression (data not shown). In contrast, the NT26/C6R2 tumors had become resistant to TSP1 (Fig. 3E) and their microvessel density was not reduced in the presence of TSP1 (Table 1). The total angiogenic activity secreted by resistant cells increased more than threefold over that secreted by parental or sensitive cells (Table 1). Although C6 cells are known to produce VEGF, in our case, studies with neutralizing antibodies showed that it is not their major angiogenic factor and indeed only a modest increase was observed in resistant cells.

Expression of TSP1 in NT26/C6 tumors induced TGF $\beta$ activation (Fig. 3D). As frequently observed in human glioblastomas (Jennings and Pietenpol 1998), neither the parental C6 nor the TSP1-sensitive C6S1 cells were growth-inhibited by TGF $\beta$. However, a growth-promoting effect of recombinant TGF $\beta$ was detected in the resistant C6R2 cells (Table 1). A transfected PAI-1 promoter reporter construct was activated to a similar ex- tent in all three clones by TGF- $\beta 1$, indicating that all expressed a functional TGF $\beta$ type II receptor (Fig. 3F).

\section{Discussion}

In this work, we have provided evidence, using two different tumor models, a fibrosarcoma and a glioblastoma, that TSP1 expression in the tumor bed postpones the onset of tumor growth and decreases initial growth rate. This delay resulted at least in part from a reduction in blood supply due to the anti-angiogenic activity of TSP1. Despite the continuous presence of TSP1, tumors eventually grew progressively, becoming resistant to its effects. Resistant tumor cells markedly increased their secretion of angiogenic factors, counterbalancing inhibitory effects of TSP1. In the fibrosarcoma model this was due in part to the increased secretion of VEGF. Interestingly, endostatin therapy also causes in situ a compensatory increase of VEGF production in mammary tumors 
(Ding et al. 2001). In both models TSP1-resistant cells also grew more effectively in the presence of TGF $\beta$, a feature frequently observed during tumor progression (Wieser 2001).

Although a large number of studies already demonstrate the usefulness of anti-angiogenic agents in experimental and clinical settings, to the best of our knowledge, this is the first report demonstrating acquired tumor resistance to an anti-angiogenic agent. In fact, previous reports where drugs were delivered exogenously at discrete intervals and tumors were allowed to re-grow in the absence of drug have stated that tumors did not develop resistance to AGM-1470 (Brem et al. 1994) or endostatin (Boehm et al. 1997; Kerbel 1997). Such protocols clearly demonstrate what they have been designed to show, namely that the normal endothelial cells do not become resistant to anti-angiogenic agents. But by allowing tumors to recover in the absence of drug, they do not aggressively apply continuous selection pressure to the growing tumor cells themselves. In contrast, we delivered the anti-angiogenic agent continuously to the tumor bed, placing the injected tumor cells under constant selective pressure. This may be the reason we were able to observe the emergence of resistant tumor cells. These tumor-resistant cells secreted increased quantities of survival factors that were apparently sufficient to protect endothelial cells from the effects of anti-angiogenic TSP1. In addition, the multiplicity of anti-tumor activities associated with the large TSP1 molecule (Bein and Simons 2000; Bornstein 1995) may make it an unusually potent selective agent.

Like all tumor populations, the C6 and JT cells used here are very heterogeneous. Even subcloning the initial clones containing the tet constructs revealed that they were composed of cells that varied in growth rate and in amounts of TSP1 that dox could induce. This heterogeneity persisted in vivo for, in tumor sections, highly vascularized regions could be observed contiguous to areas of lesser vascularization demonstrating variability in angiogenic properties of the tumor cells in situ, as is often seen also in human tumors. The development of TSP1 resistance in such a mixed population of cells could have several causes. It could be that TSP1 induces a mutation conferring a growth advantage to a cell that would be selected by clonal selection. The high percentage of resistant cells in fibrosarcoma and glioblastoma tumors is not compatible with this hypothesis. Or it could be that multiple distinct clones that are already present in the population and that, for a variety of reasons, are more angiogenic and somewhat more resistant to TGF $\beta$ grow out in the presence of TSP1. The latter hypothesis of polyclonal selection is favored because it can be demonstrated in vitro that clones with the characteristics of TSP1-resistant tumor cells pre-exist in the parental populations. Cells resistant to TGF $\beta$ could be seen when parental cells were grown in low serum containing this cytokine. Moreover, clones able to form tumors in the presence of TSP1 pre-exist. When JT8 was used in a tail vein assay in the absence of dox, some lung tumors formed, representing about $1 \%$ of those that formed in the absence of TSP1 or when resistant cells were tested. We thus propose that TSP1 counter-selects tumor cells that do not thrive in its presence. Some of the resistant cells are more angiogenic and others grow in the presence of TGF $\beta$, while still others could have undergone additional undetected changes while expanding in the presence of multifonctional TSP1. We have shown in this study that the more the initial population is heterogeneous, the higher is the percentage of emerging resistant cells. Synergistic helper effects were observed between resistant cells: mixing three fibrosarcoma clones, each containing $10 \%$ of resistant cells, resulted in the emergence of more than $50 \%$ of cells able to bypass TSP 1 inhibition. Non-resistant tumor cells also favor the growth of TSP1-resistant ones: when TSP1 was turned on after $18 \mathrm{~d}$ in tumors, when cells had divided about 12 times and the heterogeneity of the cell population had thus increased, resistance developed more rapidly than when TSP1 was expressed starting on day 0. Multiple clonal variants would cooperate during tumor progression through "community" effects (Jouanneau et al. 1999) involving secreted factors, cell-cell interactions, or both. Resistance to TSP1, as it results among others in an increased secretion of angiogenic factors, would be one of the mechanisms involved in these community effects. The percentage of cells able to grow as metastases in the presence of TSP $1(1 \%)$, which is much lower than the percentage of resistant cells in subcutaneous JT8 tumors (about 10\%), suggests that a single cell must have accumulated more resistant traits to grow when it is isolated than when it grows in the vicinity of other tumor cells. This demonstrates that resistance to TSP1 can occur even in the absence of community effects.

Those human tumors where high levels of TSP1 are associated with a poor prognosis may have overcome the effects of TSP1 in ways similar to those demonstrated here for JT sarcoma and C6 glioma. The protocol that we followed quite closely mimics the conditions likely to occur in naturally-arising human tumors that develop despite their continuous production in the tumor bed of high levels of inhibitors of angiogenesis (Chen et al. 1995; Ohtani et al. 1999; Volpert et al. 1998; Yamashita et al. 1998). Thus our results strongly support the idea that antiangiogenic agents will ultimately be most effective when used in conjunction with cytotoxic therapies, not only because of their demonstrated synergistic action in killing endothelial cells (Kerbel et al. 2000), but also because they directly reduce tumor burden and thus lessen the possibility that resistant tumor cells will emerge.

\section{Materials and methods}

Cell culture, transfections, Northern blotting, and conditioned media

Cells were grown in DMEM (GIBCO) supplemented with $10 \%$ fetal calf serum (Dutscher D10F). JT4 and JT8 cells were grown in D10F containing $50 \mu \mathrm{g} / \mathrm{mL}$ hygromycin (Boehringer Mannheim), $2 \mu \mathrm{g} / \mathrm{mL}$ puromycin (Sigma), and $100 \mathrm{ng} / \mathrm{mL}$ doxycycline (dox, Sigma); NT26 in D10F containing $0.8 \mu \mathrm{g} / \mathrm{mL}$ puromycin, $0.8 \mathrm{mg} / \mathrm{mL}$ G418 (GIBCO), and $2 \mathrm{ng} / \mathrm{mL}$ dox. Northern blot 
analysis of TSP1 and luciferase mRNA was performed as described previously (Dejong et al. 1999). S26 is a ribosomal mRNA used as a loading control. To collect conditioned media $(\mathrm{CM})$, the cells were grown for $5 \mathrm{~d}$ in complete media, rinsed twice for 10 min in DMEM, and incubated for 3 additional $\mathrm{d}$ in serum-free medium. CM were collected, protease inhibitors (PMSF, $100 \mu \mathrm{M}$ ) added except for angiogenesis assays, and CM were concentrated 25 -fold and dialyzed against PBS using Centricon-3 (Millipore) concentrators. When stated, human recombinant TGF $\beta 1$ (GIBCO), $200 \mathrm{pM}$ final concentration, or vehicle (5 mM HCl, 90.1\% BSA) was added to the culture media.

\section{TSP1-inducible cell lines}

pBiL/TSP1 bidirectional vector was constructed by inserting a fragment containing the entire mouse TSP1 coding sequence, released from a CMV expression vector (gift of V. Dixit, University of Michigan School of Medicine, Ann Arbor) in the pBiL plasmid (Clontech). Vector encoding tet-VP16 chimera was cotransfected in cJ4 or in NIH3T3 cell lines with another plasmid conferring hygromycin (c)4) or puromycine (NIH3T3) resistance. Stable transfectants were selected and cotransfected with the pBiL/TSP1 construct and a vector conferring puromycin (cJ4) or neomycine (NIH3T3) resistance. Luciferase induction upon dox withdrawal was used to seek inducible clones among the stable transfectants. A control cell line was obtained by introduction in cJ4/tet-VP16 cells of a bidirectional tet-inducible vector encoding for luciferase only. During clonal selection, TSP1 expression was suppressed by constant addition of dox to impair activation of latent TGF $\beta$ that could modify the response of the tumor cells to this cytokine.

\section{Immunoblotting}

Western blot analysis for TSP1 content was performed on concentrated conditioned media resolved by electrophoresis through a $7 \%$ SDS-polyacrylamide gel as described previously (Dejong et al. 1999) using the anti-TSP1 antibody Ab-4 (clone A6.1, NeoMarkers), diluted to $1 / 200$.

\section{Tumorigenicity assays}

JT8 cells were grown in the presence of $50 \mathrm{ng} / \mathrm{mL}$ dox (Sigma), harvested, suspended in PBS, and injected subcutaneously $\left(10^{6}\right.$ cells/site) into the hind quarters of 6-wk-old female syngeneic Fisher rats or athymic Swiss mice (5-7 animals per group). Alternatively, C6 cells were grown, harvested, and mixed with NT26 fibroblasts grown in the presence of dox. A mix of $10^{5}$ C6 and $10^{6}$ NT26 per site was injected into nude mice. When stated, $100 \mathrm{mg} / \mathrm{mL}$ dox was added in treated groups to the drinking supply of the animals $\left(5 \%\right.$ sucrose in $\mathrm{H}_{2} \mathrm{O}$, replenished twice a week). The size of the tumors was determined at scheduled intervals by external measurements of the tumors in two dimensions with a caliper. Volume (V) was estimated as $\mathrm{V}=\mathrm{Lxl}^{2}$, where $\mathrm{L}$ is the widest diameter and 1 the smallest. The accuracy of the calculations was checked comparing the calculated volume and the weight of the tumor at harvesting point. Metastasis experiments were performed injecting $10^{6}$ cells in the tail vein of athymic swiss mice (12 animals). Half of those received a dox supply after the injection while the other half received no treatment. At the end of the experiments $(3 \mathrm{wk}$ for metastasis experiments) animals were sacrificed and the tumors or lungs dissected. One-half was immediately fixed in Bouin's solution (Sigma). The other half was either frozen at $-80^{\circ} \mathrm{C}$ or trypsinized to collect cells for growth in culture. The care of animals was provided by the staff of the animal quarters of the CNRS Institut
André Lwoff in Villejuif (SEAT) according to the institutional guidelines.

\section{Immunohistochemistry}

Bouin's or formaldehyde-fixed, paraffin-embedded tumor sections (5 $\mu \mathrm{m}$ thick) were deparaffinized, rehydrated, and immunostained using automated immunostaining apparatus (NexES, Ventana, Strasbourg, France) with standardized duration and temperature of all the steps. For TSP1 detection, tissue sections were pretreated for $10 \mathrm{~min}$ in phosphate-buffered saline (PBS) containing $0.2 \%(\mathrm{v} / \mathrm{v})$ Tween 20 and $0.1 \%$ BSA, then for $10 \mathrm{~min}$ in PBS/BSA containing $0.3 \%(\mathrm{v} / \mathrm{v})$ Tween 20 . Endogenous peroxidases were quenched in $1.5 \%(\mathrm{v} / \mathrm{v})$ hydrogen peroxide for 15 min, 5 min rinse in PBS/BSA containing $0.2 \%$ Tween 20, and a brief rinse in tap water. After pretreatment, sections were incubated for $32 \mathrm{~min}$ at $37^{\circ} \mathrm{C}$ in PBS/BSA containing $0.2 \%(\mathrm{v} / \mathrm{v})$ Triton X-100 and $13 \mu \mathrm{g} / \mathrm{mL}$ of anti-TSP1 mouse monoclonal antibody, Ab-8 (NeoMarkers). For microvessel detection, tissue sections were microwave-heated $(20 \mathrm{~min}, 400 \mathrm{~W})$ and incubated for $32 \mathrm{~min}$ at $37^{\circ} \mathrm{C}$ in PBS/BSA with a $1 / 150$ dilution of a goat anti-mouse antibody, CD31 (Santa Cruz) to label endothelial cells. The remainder of the procedure was carried out as described in Clézardin et al. (1993). Immunolabeling of TGF $\beta$ was performed using the MAB1835 from R\&D (formerly clone 1D11.16 from Genzyme), which recognizes only the active forms of TGF $\beta 1,2$, and 3 as described (Crawford et al. 1998).

\section{Scoring of the microvessel density}

Highly vascularized regions of the invasive tumors ("hot spots") were selected at low magnification (10X objective). For each tumor, microvessel counts of hot spots were performed in three nonoverlapping high power fields (HPF) (40X objective; area $0.283 \mathrm{~mm}^{2}$ ). The microvessel score for each tumor was expressed as the mean of counts obtained in at least three separated $\mathrm{HPF}$.

\section{Angiogenesis assay}

In vitro endothelial cell migrations were performed as described previously (Volpert et al. 1998) in a modified Boyden chamber where cells migrated from the lower to the upper well. DME, supplemented with $0.1 \%$ BSA, was used as a negative control and $10 \mathrm{ng} / \mathrm{mL}$ bFGF as a positive control. All samples were tested in quadruplicate. Data presented were chosen from at least two representative experiments, and statistically evaluated using two-tailed Student's T-test. Bovine adrenal capillary endothelial cells BP10T8 at passages 13-14 (a gift of Dr. J. Folkman, Children's Hospital, Harvard Medical School, Boston) were used in the assay and maintained in DME supplemented with 10\% donor calf serum (JHR Biosciences, Lenexa, KS), 100 $\mu \mathrm{g} / \mathrm{mL}$ endothelial cell mitogen (Biomedical Technologies, Inc., Stoughton, MA), 2 mM glutamine. TSP1 was neutralized with monoclonal antibody A4.1 (Volpert et al. 1998) and TGFß with MAB1835 from R\&D. Results are expressed as the percentage of a maximal migration where migration toward $10 \mathrm{ng} / \mathrm{mL}$ bFGF in assay medium is arbitrarily set as $100 \%$, after subtraction of the background migration in the presence of BSA alone. Human TSP1 was purified from platelets as described (Volpert et al. 1998).

\section{Immuno assay for VEGF}

VEGF in tumor CM was measured using ELISA kit for rodent protein (R\&D Systems) according to manufacturer's instruc- 
tions. Serial dilutions of each medium were tested in triplicate, the points on the linear part of the calibration curve chosen and used to calculate average values and standard errors. The measurements were taken at least twice for each CM.

\section{TGFß-reporter vector assays}

Cells were transfected overnight with a PAI-1 luciferase reporter (p3TP-Lux [Wrana et al. 1992]) or with a PAI-1-SEAP vector, constructed inserting the Xhol-Xbal fragment of p3TPLux in pSEAP basic 2 (Clontech). After transfection, cells were split into two sister plates that were incubated for $18 \mathrm{~h}$ either in serum-free medium or in the same medium containing $5 \mathrm{ng} / \mathrm{mL}$ of recombinant human TGF $\beta 1$. SEcreted Alkaline Phosphatase (SEAP) was assayed $24 \mathrm{~h}$ later in $30 \mu \mathrm{l}$ of cell culture medium using a sensitive luminometric assay (Phosphalight, ICN). Luciferase activity was measured on cell lysates.

\section{Acknowledgments}

The authors gratefully acknowledge Marie-Pierre David and the Villejuif's SEAT animal house technicians for excellent technical help and Valérie Dejong, Annick Harel-Bellan, Slimane AitSi-Ali, and François-Xavier Barre for helpful discussions. This work was supported by grants from the Association pour la Recherche sur le Cancer (FC, PC), the Groupement des Entreprises Françaises dans la lutte contre le Cancer (FC), the Association pour la Recherche sur les tumeurs de la Prostate (FC), the Fondation pour la Recherche Médicale (FC), the National Cancer Institute, grants CA52750, CA64239 (NB), and AHA0030023N (OV). AD was supported by a fellowship from the Ligue Nationale contre le Cancer.

The publication costs of this article were defrayed in part by payment of page charges. This article must therefore be hereby marked "advertisement" in accordance with 18 USC section 1734 solely to indicate this fact.

\section{References}

Bein, K. and Simons, M. 2000. Thrombospondin type 1 repeats interact with matrix metalloproteinase 2. Regulation of metalloproteinase activity. J. Biol. Chem. 275: 32167-32173.

Bertin, N., Clézardin, P., Kubiak, R., and Frappart, L. 1997. Thrombospondin-1 and -2 messenger RNA expression in normal, benign, and neoplastic human breast tissues: Correlation with prognostic factors, tumor angiogenesis, and fibroblastic desmoplasia. Cancer Res. 57: 396-399.

Boehm, T., Folkman, J., Browder, T., and O'Reilly, M.S. 1997. Antiangiogenic therapy of experimental cancer does not induce acquired drug resistance. Nature 390: 404-407.

Bornstein, P. 1995. Diversity of function is inherent in matricellular proteins: An appraisal of thrombospondin 1. J. Cell. Biol. 130: 503-506.

Boukamp, P., Bleuel, K., Popp, S., Vormwald-Dogan, V., and Fusenig, N.E. 1997. Functional evidence for tumor-suppressor activity on chromosome 15 in human skin carcinoma cells and thrombospondin-1 as the potential suppressor. $I$. Cell. Physiol. 173: 256-260.

Brem, H., Goto, F., Budson, A., Saunders, L., and Folkman, J. 1994. Minimal drug resistance after prolonged antiangiogenic therapy with AGM-1470. Surgical forum XLV: 674677.

Campbell, S.C., Volpert, O.V., Ivanovich, M., and Bouck, N.P. 1998. Molecular mediators of angiogenesis in bladder cancer. Cancer Res. 58: 1298-1304.
Canfield, A.E. and Schor, A.M. 1995. Evidence that tenascin and thrombospondin-1 modulate sprouting of endothelial cells. I. Cell. Sci. 108: 797-809.

Chen, C., Parangi, S., Tolentino, M.J., and Folkman, J. 1995. A strategy to discover circulating angiogenesis inhibitors generated by human tumors. Cancer Res. 55: 4230-4233.

Clézardin, P., Frappart, L., Clerget, M., Pechoux, C., and Delmas, P.D. 1993. Expression of thrombospondin (TSP1) and its receptors (CD36 and CD51) in normal, hyperplastic, and neoplastic human breast. Cancer Res. 53: 1421-1430.

Crawford, S.E., Stellmach, V., Murphy-Ullrich, J.E., Ribeiro, S.M., Lawler, J., Hynes, R.O., Boivin, G.P., and Bouck, N. 1998. Thrombospondin-1 is a major activator of TGF-betal in vivo. Cell 93: 1159-1170.

Dejong, V., Degeorges, A., Filleur, S., Ait-Si-Ali, S., Mettouchi, A., Bornstein, P., Binetruy, B., and Cabon, F. 1999. The Wilms' tumor gene product represses the transcription of thrombospondin 1 in response to overexpression of c-Jun. Oncogene 18: 3143-3151.

Ding, I., Sun, J.Z., Fenton, B., Liu, W.M., Kimsely, P., Okunieff, P. and Min, W. 2001. Intratumoral administration of endostatin plasmid inhibits vascular growth and perfusion in MCa-4 murine mammary carcinomas. Cancer Res. 61: 526531.

DiPietro, L.A., Nebgen, D.R., and Polverini, P.J. 1994. Downregulation of endothelial cell thrombospondin 1 enhances in vitro angiogenesis. J. Vasc. Res. 31: 178-185.

Good, D.J., Polverini, P.J., Rastinejad, F., Le, B.M., Lemons, R.S., Frazier, W.A., and Bouck, N.P. 1990. A tumor suppressordependent inhibitor of angiogenesis is immunologically and functionally indistinguishable from a fragment of thrombospondin. Proc. Natl. Acad. Sci. 87: 6624-6628.

Gossen, M. and Bujard, H. 1992. Tight control of gene expression in mammalian cells by tetracycline- responsive promoters. Proc. Natl. Acad. Sci. 89: 5547-5551.

Iruela-Arispe, M.L., Bornstein, P. and Sage, H. 1991. Thrombospondin exerts an antiangiogenic effect on cord formation by endothelial cells in vitro. Proc. Natl. Acad. Sci. 88: 50265030.

Jennings, M.T. and Pietenpol, J.A. 1998. The role of transforming growth factor beta in glioma progression. J. Neurooncol. 36: $123-140$.

Jouanneau, J., Moens, G., and Thiery, J.P. 1999. The community effect in FGF-1 mediated tumor progression of a rat bladder carcinoma does not involve a direct paracrine signaling. Oncogene 18: 327-333.

Kerbel, R.S. 1997. A cancer therapy resistant to resistance. $\mathrm{Na}$ ture 390: 335-336.

Kerbel, R.S., Viloria-Petit, A., Klement, G. and Rak, J. 2000. 'Accidental' anti-angiogenic drugs. Anti-oncogene directed signal transduction inhibitors and conventional chemotherapeutic agents as examples. Eur. I. Cancer 36: 12481257.

Massague, J., Blain, S.W., and Lo, R.S. 2000. TGFbeta signaling in growth control, cancer, and heritable disorders. Cell 103: 295-309.

Mettouchi, A., Cabon, F., Montreau, N., Vernier, P., Mercier, G., Blangy, D., Tricoire, H., Vigier, P., and Binetruy, B. 1994. SPARC and thrombospondin genes are repressed by the c-jun oncogene in rat embryo fibroblasts. EMBO J. 13: 5668-5678.

Morelli, D., Lazzerini, D., Cazzaniga, S., Squicciarini, P., Bignami, P., Maier, J.A., Sfondrini, L., Menard, S., Colnaghi, M.I., and Balsari, A. 1998. Evaluation of the balance between angiogenic and antiangiogenic circulating factors in patients with breast and gastrointestinal cancers. Clin. Cancer Res. 4: $1221-1225$. 
Ohtani, Y., Kijima, H., Dowaki, S., Kashiwagi, H., Tobita, K., Tsukui, M., Tanaka, Y., Tsuchida, T., Tokunaga, T., Yamazaki, H., et al. 1999. Stromal expression of thrombospondin-1 is correlated with growth and metastasis of human gallbladder carcinoma. Int. J. Oncol. 15: 453-457.

O'Reilly, M.S., Holmgren, L., Shing, Y., Chen, C., Rosenthal, R.A., Moses, M., Lane, W.S., Cao, Y., Sage, E. H., and Folkman, J. 1994. Angiostatin: A novel angiogenesis inhibitor that mediates the suppression of metastases by a Lewis lung carcinoma. Cell 79: 315-328.

Prehn, R.T. 1993. Two competing influences that may explain concomitant tumor resistance. Cancer Res. 53: 3266-3269.

Salnikow, K., Cosentino, S., Klein, C., and Costa, M. 1994. Loss of thrombospondin transcriptional activity in nickel-transformed cells. Mol. Cell. Biol. 14: 851-858.

Sheibani, N. and Frazier, W.A. 1995. Thrombospondin 1 expression in transformed endothelial cells restores a normal phenotype and suppresses their tumorigenesis. Proc. Nat1. Acad. Sci. 92: 6788-6792.

- 1996. Repression of thrombospondin-1 expression, a natural inhibitor of angiogenesis, in polyoma middle $T$ transformed NIH3T3 cells. Cancer Lett. 107: 45-52.

Slack, J.L. and Bornstein, P. 1994. Transformation by v-src causes transient induction followed by repression of mouse thrombospondin-1. Cell. Growth Differ. 5: 1373-1380.

Streit, M., Velasco, P., Brown, L.F., Skobe, M., Richard, L., Riccardi, L., Lawler, J., and Detmar, M. 1999. Overexpression of thrombospondin-1 decreases angiogenesis and inhibits the growth of human cutaneous squamous cell carcinomas. Am. J. Pathol. 155: 441-452.

Tikhonenko, A.T., Black, D.J., and Linial, M.L. 1996. Viral Myc oncoproteins in infected fibroblasts down-modulate thrombospondin-1, a possible tumor suppressor gene. J. Biol. Chem. 271: 30741-30747.

Tolsma, S.S., Stack, M.S., and Bouck, N. 1997. Lumen formation and other angiogenic activities of cultured capillary endothelial cells are inhibited by thrombospondin-1. Microvasc. Res. 54: 13-26.

Tolsma, S.S., Volpert, O.V., Good, D.J., Frazier, W.A., Polverini, P.J., and Bouck, N. 1993. Peptides derived from two separate domains of the matrix protein thrombospondin-1 have antiangiogenic activity. J. Cell. Biol. 122: 497-511.

Volpert, O.V., Lawler, J., and Bouck, N.P. 1998. A human fibrosarcoma inhibits systemic angiogenesis and the growth of experimental metastases via thrombospondin-1. Proc. Nat1. Acad. Sci. 95: 6343-6348.

Weinstat-Saslow, D.L., Zabrenetzky, V.S., VanHoutte, K., Frazier, W.A., Roberts, D.D., and Steeg, P.S. 1994. Transfection of thrombospondin 1 complementary DNA into a human breast carcinoma cell line reduces primary tumor growth, metastatic potential, and angiogenesis. Cancer Res. 54: 6504-6511.

Wieser, R. 2001. The transforming growth factor-beta signaling pathway in tumorigenesis. Curr. Opin. Oncol. 13: 70-77.

Wrana, J.L., Attisano, L., Carcamo, J., Zentella, A., Doody, J., Laiho, M., Wang, X.F., and Massague, J. 1992. TGF beta signals through a heteromeric protein kinase receptor complex. Cell 71: 1003-1014.

Yamashita, Y., Kurohiji, T., Tuszynski, G.P., Sakai, T., and Shirakusa, T. 1998. Plasma thrombospondin levels in patients with colorectal carcinoma. Cancer 82: 632-638.

Zabrenetzky, V., Harris, C.C., Steeg, P.S., and Roberts, D.D. 1994. Expression of the extracellular matrix molecule thrombospondin inversely correlates with malignant progression in melanoma, lung and breast carcinoma cell lines. Int. J. Cancer 59: 191-195. 




\section{In vivo mechanisms by which tumors producing thrombospondin 1 bypass its inhibitory effects}

Stéphanie Filleur, Olga V. Volpert, Armelle Degeorges, et al.

Genes Dev. 2001, 15:

Access the most recent version at doi:10.1101/gad.193501

References This article cites 40 articles, 20 of which can be accessed free at: http://genesdev.cshlp.org/content/15/11/1373.full.html\#ref-list-1

License

Email Alerting

Receive free email alerts when new articles cite this article - sign up in the box at the top Service right corner of the article or click here.

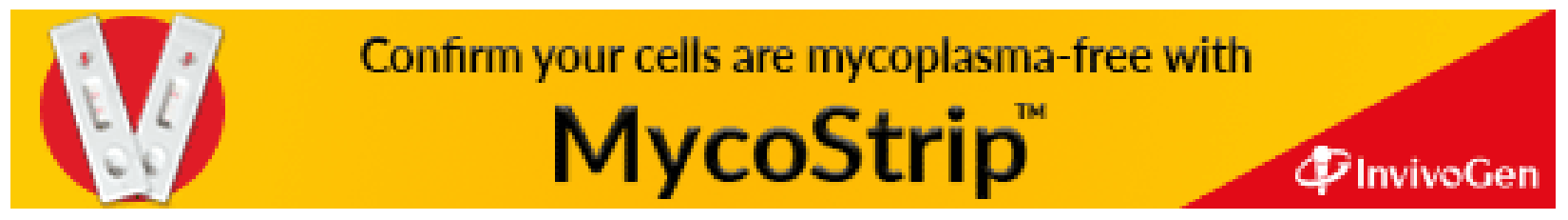

